\title{
Problemas metodológicos en las investigaciones sobre VIH/SIDA en Bolivia
}

\author{
Methodological problems \\ in the scientific research on HIV /AIDS in Bolivia
}

\begin{abstract}
This paper discusses the methodological problems in the scientific research on HIVI AIDS in Bolivia, both in the areas of epidemiology and social sciences. Studies associated with this research served as the basis for the implementation of health programs run by The Global Fund, The Pan-American Health Organization, International Cooperation, Non-Governmental Organizations and the Bolivian Ministry of Health and Sports. An analysis of the methodological contradictions and weaknesses was made by reviewing the bibliography of the studies and by conducting qualitative methodological research, that was focused on the quality of health care available to people living with HIV/AIDS in public hospitals and health centers, and looked at how programs targeted at this sector of the population are designed and delivered. In this manner, it was possible to observe the shortcomings of the methodological design in the epidemiological and social science studies which serve as the basis for the implementation of these health programs.
\end{abstract}

Key words Methods, epidemiology, HIV/AIDS, Bolivia
Resumen Este trabajo es una reflexión sobre las dificultades metodológicas que arrastra la producción científica, tanto epidemiológica como de ciencias sociales, relativa a la problemática del VIH/ SIDA en Bolivia. Los estudios asociados a esta producción sirvieron de base para la implementación de programas del Fondo Mundial, la Organización Panamericana de la Salud, cooperaciones internacionales, Organizaciones No Gubernamentales y el Ministerio de Salud y Deportes boliviano. El análisis de las contradicciones y falencias metodológicas se realizó a través de una revisión bibliográfica y una investigación de metodología cualitativa, que se centró en la calidad de atención a las personas viviendo con VIH/SIDA en servicios públicos de salud y en cómo son realizados y diseñados los programas destinados a esta población. De esta manera se pudo observar las deficiencias en los diseños metodológicos que presentan los estudios epidemiológicos y de ciencias sociales que sirven de base para la implementación de programas sanitarios.

Palabras clave Métodos, Epidemiología, VIH/ SIDA, Bolivia
${ }^{1}$ Departamento de Antropología, Facultad de

Ciencias Sociales y Jurídicas, Universidad de Tarapacá. 18 de Septiembre 2222. 1000000 Arica Chile. susanahita@yahoo.es 


\section{Introducción}

Este trabajo tiene su origen en una investigación realizada durante los años 2009/2010 en la región del oriente Boliviano, en las ciudades de Cobija, Trinidad y Santa Cruz, sobre la calidad de atención en los servicios públicos de salud a las personas que viven con VIH/SIDA.

A pesar de que los antirretrovirales ya se administraban de manera gratuita en el país, a lo largo de los años 2008 y 2009 se produjo un incremento de la mortalidad. Ante este aumento, se inició la investigación intentando entender los factores que envolvían a la población afectada en las zonas menos atendidas por el gobierno central.

En esa investigación se hizo un total de 90 entrevistas a personal sanitario, personas viviendo con VIH/SIDA (PVVS), familiares o acompañantes de PVVS y/o fallecidas, y representantes de asociaciones u Organizaciones No Gubernamentales que trabajan sobre VIH/SIDA. También se realizó observación en los tres hospitales públicos más importantes de esas ciudades y en los servicios públicos de salud que atienden a este tipo de población, denominados Centros de Vigilancia y Referencia (CDVIR). Los datos epidemiológicos que mostraban la patología en el país comenzaron a tomar otra dimensión a partir de la realización del trabajo de campo, en el que me interioricé en cómo son recogidos estos datos, en qué planillas se transcriben, quiénes las diseñan y financian y, por ende, cómo son analizadas.

Este artículo pretende abrir la reflexión en torno a los problemas metodológicos que arrastran las investigaciones realizadas en Bolivia sobre VIH/ SIDA, así como las estrategias de investigación y las razones o causas de por qué se adoptan.

El primer caso de Sida en Bolivia se registró en la ciudad de Santa Cruz en el año 1984, y en el 2009 el número de casos notificados era de 4.889. Sobre estos últimos, hasta diciembre del 2009 se contabilizaban 498 defunciones ${ }^{1}$. De los datos del informe UNGASS (sigla en inglés de la sección especial de la Asamblea General de las Naciones Unidas sobre el SIDA) relativos a los años 2006 y 2007 se desprende que el número de casos notificados fue de 498 y 467 respectivamente. La gran mayoría de éstos, el 89\%, se localiza en los departamentos de Santa Cruz, Cochabamba y La Paz, en tanto que el 11\% restante se reparte entre los otros departamentos del país. En el departamento de Santa Cruz más de la mitad de los casos, el 55\%, se concentra en el grupo de personas que va de los 15 a los 44 años y, dentro de éste, el subgrupo más afectado es el que forman las personas que tienen de 15 a 24 años ${ }^{2}$.
Bolivia ocupa, según ONUSIDA (2005) el penúltimo lugar entre los países de Sudamérica y el Caribe en cuanto a la incidencia del SIDA, con una prevalencia menor al $1 \%$ en el ámbito de la población general ${ }^{2}$. Se trata de una prevalencia calculada exclusivamente sobre los pacientes que acceden a los servicios de salud, de manera que estamos ante datos de escasa fiabilidad si se tiene en cuenta que una gran parte de la población no recurre a los centros de salud al valorar negativamente la atención que recibe en los mismos ${ }^{3,4}$. Este no es un hecho menor ya que los registros de morbimortalidad se basan únicamente en los datos registrados en el interior de los servicios de salud, existiendo, por tanto un subregistro, no sólo del VIH/SIDA sino de numerosas patologías.

En un artículo publicado en $2009^{5}$ se daba a conocer que los principales problemas de salud no eran registrados, entre otros motivos, porque no existía espacio para registrar las causas reales de mobimortalidad. Las planillas para recoger los datos se diseñan a partir de las políticas internacionales y con el objetivo de realizar comparaciones con el resto de los países de Latinoamérica y el Caribe. Es importante destacar que el Ministerio de Salud y Deportes reconoce la alta subnotificación de la mortalidad ya que, entre otros motivos, el sistema no cuenta con datos del sector privado ni de la seguridad social ${ }^{2}$. Existe, por tanto, un desconocimiento de los datos epidemiológicos reales, desconocimiento que se extiende a las diversas problemáticas propias de la epidemia en el país.

\section{La construcción de la patología del VIH/SIDA en Bolivia}

El Programa Nacional de ITS/SIDA se inició en Bolivia en el año 1986 en la oficina de Epidemiología del Ministerio de Salud y Deportes. Desde 1992 la agencia de los Estados Unidos para el Desarrollo Internacional (USAID) cooperó con el Programa Nacional de ITS/SIDA. Inicialmente, los fondos eran asignados al entonces llamado Proyecto contra SIDA y, desde 1999, por PROSIN, a través del Proyecto de Salud Integral, financiado por USAID. Desde 1986 el Programa Nacional recibió asistencia técnica de la Organización Panamericana de la Salud (OPS), USAID a través de PROSIN y de expertos del Centro para la Prevención y Control de Enfermedades de Atlanta (CDC), y a partir del año 2002 recibe asistencia técnica y financiera del Programa Nacional ITS/SIDA de Brasil ${ }^{6}$, y posteriormente del Fondo Mundial. 
En 1992 USAID creó el Proyecto contra el SIDA, que subsiguientemente pasó a denominarse Programa de Infecciones de Transmisión Sexual (ITS); a partir del año 2000 los espacios de atención del Programa cambiaron el nombre a Centro Departamental de Vigilancia y de Referencia (CDVIR).

El principal objetivo de USAID era frenar la expansión del VIH a través de las trabajadoras sexuales, al considerar que no usaban preservativo o no lo utilizaban correctamente. A su vez, pretendía habilitar laboratorios en los centros de salud a los que acudían dichas trabajadoras (recién en el año 2006 se consolidaron laboratorios en todos los CDVIR del país). En un principio, la atención médica se destinaba sólo a esta población. No se contaba con laboratorio y la calidad de atención era motivo de muchas quejas. Comenzó el control de las trabajadoras sexuales en estos servicios, que consistió en firmar un carné sanitario que les autorizaba para trabajar en ausencia de ITS y VIH. De manera que la forma en que originalmente se implementó el programa VIH/SIDA en el país situó a la prostitución como clave de la difusión y del control de la patología.

Desde su implementación, los CDVIR se destinaron fundamentalmente a trabajadoras sexuales y en menor medida a hombres que tienen sexo con hombres. Cuando la epidemia comenzó a aparecer en la población general a la que se etiquetó con el término de "heterosexual", esta población general debía atenderse en los mismos espacios, que se crearon para grupos específicos. De esta manera a la población general con VIH se le proporcionaba, como única alternativa la atención en los CDVIR, donde además de servir para el registro epidemiológico, era y es el único lugar donde los antirretrovirales se entregan gratuitamente.

Este comienzo inadecuado se mantuvo a lo largo de los años, encontrándonos actualmente con que la mayoría de la población afectada se atiende en estos servicios, cuyos especialistas encargados son ginecólogos, ya que estos centros fueron diseñados para atender a las trabajadoras sexuales mujeres. Se dio por hecho que los espacios de mayor riesgo son los lenocinios; sin embargo, las trabajadoras sexuales no sólo trabajan en lenocinios legales y autorizados, en los cuales se les obliga a tener el carné sanitario; sino que la mayoría trabaja en espacios no controlados ni autorizados.

Del querer controlar la epidemia a través de los/las trabajadores sexuales se derivó una serie de problemas. En primer lugar, el no llegar al total de personas que utilizaban el sexo como medio de trabajo. Además, lo que se consiguió fue crear un gueto de atención en un servicio altamente estigmatizado por la población. De esta manera, los afectados de esta patología, aun siendo población infantil, son tratados por ginecólogos. Ello conlleva problemas en los tratamientos antirretrovirales ya que no existen especialistas en pediatría e infectología.

Existen actualmente 9 Centros Departamentales de Vigilancia y Referencia y 3 Centros Regionales. Muchos de los CDVIR se encuentran en ciudades pequeñas en donde todo el mundo puede ver quién ingresa y sale del servicio. La población sabe que los usuarios que entran en estos centros se encuentran afectados con alguna ITS o VIH/ SIDA, o son trabajadoras sexuales que realizan su control, motivo por el que las personas no desean ser vistas en el consultorio aunque quieran realizarse la prueba de laboratorio del VIH.

Todas estas circunstancias dificultan obtener un buen registro epidemiológico, ya que tan sólo las personas sospechosas son derivadas a los CDVIR y no todos llegan a realizarse la prueba confirmatoria, algunas desaparecen por el camino, deciden irse del país o se instalan en la zona rural.

La falta de recursos, tanto materiales como humanos, es una de las causas de la deficiente calidad que se proporciona en los servicios públicos de salud. A ello se suma que desde el tercer año de la carrera de medicina los estudiantes tratan y atienden a los pacientes en los hospitales, la mayoría de las veces sin supervisión de sus profesores. La existencia de numerosas universidades privadas con las carreras de ciencias de la salud (medicina, enfermería, auxiliar de enfermera, etc.), muchas veces sin regulación, hace que en los hospitales se encuentren más estudiantes que pacientes, motivando todo ello negligencias hospitalarias y graves problemas de atención.

Este fenómeno, sumado a la existencia de cementerios clandestinos en el país, y a que ciertas causas de muerte - suicidios, accidentes, alcoholismo, etc. -, se recojan en los registros de la policía, motiva que los datos se encuentren dispersos y no formen parte de los registros epidemiológicos ${ }^{5}$. Y hay que tener en cuenta que, por las deficientes condiciones de atención y calidad de los servicios públicos de salud buena parte de la población se autoatiende o recurre a sistemas médicos diferentes al biomédico. A lo que se añade que las clínicas privadas y la seguridad social no tienen la obligación de reportar datos de morbimortalidad a los registros nacionales y que la base de datos del Sistema de Vigilancia del Pro- 
grama ITS/VIH/SIDA no se socializa al Servicio Nacional de Información Estadística (SNIS): "Debido a la falta de funcionamiento de los subcomités de vigilancia de ITS/SIDA" 7 .

\section{Globalización y datos epidemiológicos}

La globalización en el área de la salud se insertó a partir de la implementación de lineamientos políticos internacionales que conllevaron estudios diseñados por las agencias globales de desarrollo. Se debe destacar que fueron estas agencias las que implementaron planes sobre VIH/ SIDA cuando, aún no se había despertado el interés nacional frente a esta problemática de salud.

El hecho de acotar la epidemia a lo que consideraron "grupos de riesgo" ${ }^{-10}$, hizo que las encuestas diseñadas para la vigilancia epidemiológica sobre el VIH/SIDA y el conocimiento sobre las prácticas de la población en torno a la sexualidad se realizaran para cierto tipo de poblaciones y no para la población general: trabajadoras sexuales y sus clientes, hombres que tienen sexo con hombres y usuarios de drogas intravenosas.

En Bolivia se decidió, a priori, quiénes conformaban las poblaciones de riesgo, antes de investigar donde estaba concentrada la epidemia. La mayoría de los estudios fueron financiados por la cooperación internacional y fundamentalmente por las Agencias de Naciones Unidas, USAID y el Fondo Mundial. Las primeras investigaciones sustentaron las siguientes y así se construyó una realidad apoyada en datos numéricos, a partir de estudios realizados en periodos cortos acordes al concepto de productividad imperante en las investigaciones basadas en parámetros de costo/ beneficio (investigaciones cortas e intervenciones rápidas) y, hechas con metodología cuantitativa y en algunos casos con la inclusión de técnicas cualitativas. Estas investigaciones se caracterizan por la ausencia de discusión teórica y de construcción de categorías para el contexto histórico boliviano.

Hay que tener en cuenta que la demanda de evaluación sobre los resultados de VIH/SIDA proviene de organizaciones internacionales que apoyan financieramente el Programa de ITS/VIH/ SIDA, tanto en la entrega de antirretrovirales, como en la vigilancia epidemiológica y en programas de prevención. Dado que estos organismos suelen precisar resultados numéricos, se otorga preferencia a los estudios cuantitativos que pretenden incluso contabilizar los "comportamientos, actitudes y prácticas”. El 84\% del presupuesto global nacional destinado a políticas y programas de VIH/SIDA es de cooperación in- ternacional, mientras que sólo el $16 \%$ es contribución del gobierno ${ }^{6}$. De esta dependencia financiera se deriva la supeditación de las actuaciones a criterios marcados en el exterior del país mientras que, de manera paralela, no se cuestiona la falta de rigurosidad de los estudios efectuados en plazos breves de tiempo y sin construcción teórica. Como plantea Sassen ${ }^{11}$ : "El Estado es uno de los dominios institucionales estratégicos donde se realizan las labores esenciales para el crecimiento de la globalización", por lo tanto, "el Estado no es una 'victima' de los procesos globales sino que participa en su implementación"11.

Un gran número de investigaciones en America Latina se gestaron en base a un manual que realizó Family Health Internacional en el año 2000, titulado "Encuestas de Vigilancia del Comportamiento. Directrices para encuestas del comportamiento repetidas en poblaciones en riesgo al VIH" (Manual financiado por USAID y el Departamento para el Desarrollo Internacional del Reino Unido [DFID]). El objetivo de los estudios realizados en base a este manual fue el de crear sistemas confiables de vigilancia bajo un enfoque cuantitativo. Cabe señalar que este manual facilitó pensar la problemática del VIH/SIDA en Bolivia y ayudó a planificar estudios. Sin embargo, el principal objetivo era el de responder a los requisitos internacionales, tal y como se reconoce cuando se afirma que "aun cuando deben responder a las necesidades locales, deben también en lo posible adaptarse a los estándares internacionales acordados, de tal manera que el progreso pueda ser comparado, no solamente en el tiempo sino además entre poblaciones" ${ }^{12}$. Los indicadores esenciales para cada subgrupo de población los diseñó en este estudio ONUSIDA y OPS/ OMS entre otras organizaciones.

La guía es una herramienta útil y completa que consta de todas las etapas necesarias para estudios de comportamiento sobre VIH/SIDA: selección de los grupos, abordaje del muestreo, utilización de los cuestionarios, análisis e interpretación de los resultados y utilización de los datos para mejorar la prevención del VIH, con ejemplos concretos. Pero, pese a la voluntad manifestada, no se tuvo en cuenta, sin embargo, que no todos los países poseen los medios (técnicos, científicos y financieros) para realizar seriamente las adaptaciones locales necesarias.

Posterior a esta guía en el año 2009 se difundió un documento con las Directrices para el Desarrollo de Indicadores Básicos realizado por ONUSIDA, donde se dan a conocer los indicadores básicos para medir la eficacia a la respuesta 
del VIH/SIDA. Este manual ofrece instrucciones para preparar los informes regionales que posteriormente se incorporan a los informes mundiales sobre los progresos realizados en la aplicación de la Declaración del compromiso sobre VIH/SIDA de la Asamblea General de Naciones Unidas (UNGASS). Estos indicadores básicos se agrupan en: "acciones y compromisos nacionales; conocimiento y comportamiento nacionales; impacto nacional y; compromiso y acción mundiales"13. Lo positivo de la exigencia del informe es que obliga a las autoridades de salud a reflexionar sobre la situación en la que se encuentra el país frente a la epidemia. Si bien, el objetivo es el de identificar problemas y recomendar acciones $^{13}$, la base de este informe sigue sustentándose en encuestas demográficas y de comportamiento, cuyas preguntas están basadas en la guía de Family Health International, la que encasillaba a priori la epidemia entre los gays, bisexuales $y$ trans (GBT), y secundariamente tomaba en cuenta a los hombres que tienen sexo con hombres (HSH), aconsejando realizar estudios de vigilancia epidemiológica de segundo nivel sobre estos grupos.

En el año 2009 siguiendo los cuestionarios estandarizados de comportamiento para VIH que realizó Family Health International y AIDSKAPCDC en el año 2000, y en las directrices para el desarrollo de indicadores básicos que realizó ONUSIDA $^{13}$ se concretaba un estudio de vigilancia epidemiológica de segundo nivel titulado: Estudio sobre sexualidad masculina y VIH en Bolivia (SEMVBO), que fue la base de las medidas tomadas por el Fondo Mundial para la 9na. Ronda (El estudio fue financiado por la Agencia para el Desarrollo Internacional de los Estados Unidos [USAID]; Centro para la Prevención y Control de Enfermedades [CDC]; Programa Conjunto de las Naciones Unidas sobre el VIH/SIDA [ONUSIDA]; Organización Panamericana de la Salud [OPS]; Centro de Investigación de la Marina de Estados Unidos [NMRCD-Lima] y el Fondo Mundial). La investigación, realizada en cuatro meses en las tres ciudades más pobladas de Bolivia (La Paz, Santa Cruz y Cochabamba), pretendía comprender el comportamiento de esta población frente a las ITS, el VIH/SIDA y determinar la prevalencia e incidencia del VIH y la seroprevalencia de sífilis, Herpes Virus Tipo 2. Y, a partir de este estudio, caracterizar la epidemia del VIH en el país y promover políticas y estrategias de prevención para los $\mathrm{HSH}^{14}$. Sus resultados llevaron a proponer las líneas de actuación de la 9na. Ronda del Fondo Mundial, que terminó enfocándose exclusivamen- te en esta población, GBT. El estudio sirvió para que $1200 \mathrm{HSH}$ y GBT accedieran a realizarse las pruebas de laboratorio, ya que una vez efectuada la encuesta se pasaba a la recolección de las muestras biológicas, una muestra de sangre para el estudio de sífilis, Herpes tipo 2 (HSV-2) y VIH, y un examen físico de ITS.

El cuestionario empleado en esta investigación, en el que participaron 1.019 personas, introducía una contextualización local del lenguaje empleado (cómo recomendaba la guía), pero no del contenido de las preguntas. En las tres ciudades se empleó un mismo cuestionario, sin referencia al contexto sociocultural. Las preguntas sobre comportamientos sexuales que utilizó este estudio son las siguientes:

1) ¿Puede reducirse el riesgo de transmisión del VIH manteniendo relaciones sexuales con una única pareja fiel y no infectada?

2) ¿Puede reducirse el riesgo de transmisión del VIH usando preservativo?

3) ¿Puede una persona de aspecto saludable tener VIH?

4) ¿Se puede contraer el VIH por picadura de mosquito?

5) ¿Se puede contraer el VIH compartiendo alimentos con una persona infectada?

La encuesta se realizó en un cuestionario de formato preestablecido de respuesta cerrada (si/no).

En los estudios realizados en el país, financiados por agencias globales, se formulan las mismas preguntas, referidas a poblaciones distintas; es decir, no están pensadas para el contexto y población a la que se enfoca, sino que están elaboradas desde una mirada global que decide lo que debe ser preguntado y cómo, repercutiendo todo ello en resultados poco fiables.

Es ingenuo pensar que, a través de este tipo de preguntas, planificadas para obtener resultados exclusivamente numéricos, se puedan conocer los comportamientos sexuales y los "conocimientos" que poseen los GBT con respecto al VIH. Finalmente, el estudio SEMBVO concluye, en base a estos datos, que es posible conocer el comportamiento de los hombres que mantienen sexo con otros hombres y, que este grupo poblacional es uno de los más afectados por la epidemia. Todo ello para determinar que debe fortalecerse el uso del condón, que se reconoce como poco usado.

A partir de este estudio, la propuesta de los principales indicadores de comportamiento de la población de HSH influyó en la toma de decisiones que ha concluido en los lineamientos actuales, que afecta a toda la población asentada en 
territorio boliviano y que, dado que este estudio fue retomado por el informe UNGASS, sigue determinando las políticas a adoptar por el país.

El estudio UNGASS ${ }^{1}$ (2008-2009) que se realizó con posterioridad al estudio SEMVBO, sistematizó la bibliografía realizada exclusivamente por las agencias globales basándose en los estudios anteriores $^{1}$ (Los estudios son: "SEMVBO, Prevalencia del VIH/SIDA en trabajadoras sexuales comerciales-PREVETS; Estudio de CAP sobre acciones de IEC del Programa Nacional ITS/VIH/ SIDA. La población Trans que se dedica al trabajo sexual, realizado por UNFPA en el 2009 y los estudios realizados de Conocimientos Actitudes y Prácticas en TS y HSH en el año 2008 y 2009, por el Receptor Principal del Fondo Mundial en Bolivia" ) y considerando estos estudios suficientemente científicos como para determinar las políticas a seguir. Así, el estudio UNGASS enfatiza que "el estudio SEMVBO ha permitido la caracterización de la epidemia en el país, mostrando la concentración de la misma en población GBT y HSH" '. Según este estudio: "la epidemia se caracteriza como concentrada y de bajo nivel. La concentración se refiere principalmente a grupos de mayor riesgo como la población GBT y otros $\mathrm{HSH}$, trabajadoras sexuales y clientes de trabajadoras sexuales. Evidencias estadísticas permiten inferir que la prevalencia en población GBT y otros HSH es de $12^{\prime} 7 \%$ en el caso de las trabajadoras sexuales es de 0 ' $40 \%$ "'. Por tanto, la información existente avala la creación de las iniciativas que promulga el programa de ITS/VIH/SIDA del Ministerio de Salud, dirigido actualmente en exclusividad a: trabajadoras sexuales, hombres que tienen sexo con hombres y Gay, Bisexual y Trans (GBT): travestí, transexual, transgénero.

Las guías diseñadas para la recolección y análisis de datos de salud, tiene su origen en la década del 60 cuando comenzaron a gestarse los RAP (Rapid Assessment Procedures) como una estrategia metodológica acorde con los tiempos y necesidades institucionales, fundamentalmente de las agencias de desarrollo y del sector salud ${ }^{15}$.

Dichas investigaciones mencionan la utilización de metodología cualitativa, pero únicamente utilizan técnicas cualitativas fuera de la esencia epistemológica que conlleva dicha metodología que no sólo requiere de tiempo prolongado y continuo en terreno sino de construcción teórica. Estas guías suelen estar diseñadas para investigadores no especializados, por ello se prescinde de análisis teórico y por tanto de rigor epistemológico que guíe la investigación. Tener información no significa que sea información adecuada y, sin ella ¿cómo se puede establecer criterios de intervención eficaces? La falta de reflexión teórica lleva a una deficiente recogida de datos y a un análisis inadecuado ya que se basa en un dato que no se contrasta con la realidad.

Sin embargo, estos datos epidemiológicos se presentan como el resultado de investigaciones científicas y que, en el futuro, servirán para nuevas investigaciones. De esta forma, se construye una cadena de información sin que se cuestionen las carencias metodológicas básicas de los estudios, la falta de rigurosidad y los sesgos en los enfoques.

\section{Contexto local y prevención}

Como hemos visto, estos estudios fueron diseñados para analizar el comportamiento de las trabajadoras sexuales, HSH y GBT, motivo por el cual son estos grupos los que aparecen como el foco de la epidemia sin que se considere la diversidad de la población (campesinos, indígenas rural/urbano, afrodescendientes, poblaciones mixtas, criollos, mestizos, etc.) en un país con 36 grupos étnicos.

Un aspecto importante a tener en cuenta refiere a que los términos de bisexual, homosexual, heterosexual o trans, no han sido definidos de acuerdo con el contexto específico boliviano ${ }^{15}$. Por ejemplo en el oriente del país es socialmente aceptado que un varón se ocupe de dos mujeres con sus respectivos hijos, que habitan en lugares diferentes.

La mayoría del personal sanitario entrevistado que atiende a personas viviendo con VIH/SIDA asegura que un gran porcentaje de las personas afectadas tuvieron contacto con un "homosexual", es decir, con un hombre que tuvo relaciones con otro u otros hombres. Sin embargo, en las entrevistas hechas a hombres, pese a haber tenido relación con otros hombres, se declaran heterosexuales. Según mi investigación, todas estas personas, de acuerdo con su autoidentificación y adscripción identitaria, entrarían dentro de la categoría de población general ya que no se consideran representados dentro de la categoría de "hombres que tienen sexo con hombres". Muchos de ellos tienen parejas mujeres estables y tampoco se identifican con la categoría de bisexual. En consecuencia, nos encontramos ante la disparidad existente entre las categorías proyectadas universalmente desde la globalización y las agencias internacionales - representativas de un modelo neoliberal y propias de un sistema de ideas determinado - y las especificidades y las prácticas propias de un país tan heterogéneo como Bolivia. 
A partir de las investigaciones existentes es difícil realizar campañas preventivas eficaces, ya que se desconoce lo más importante: el contenido y la forma de entender la sexualidad, el riesgo, las relaciones hombre-mujer, hombre-hombre, mujermujer, transexual, etc., y los factores que están influyendo en esta enfermedad/padecimiento que no son exclusivamente epidemiológicos sino socioculturales, ideológicos y político-económicos. Conocer o tener información no significa incorporar ni modificar prácticas, es decir, conocer que el preservativo es un método preventivo no significa que se use, aunque se conozca. Fumar provoca cáncer, es de conocimiento general de la población, sin embargo sigue habiendo fumadores por factores culturales, individuales, opcionales, ya que se asume el riesgo de manera diferenciada, ya sea grupal o individualmente.

Bolivia presenta particularidades respecto al resto de los países latinoamericanos ya que la mayor parte de su población es indígena, aspecto que no se tuvo en cuenta en las investigaciones realizadas hasta el momento.

En un comienzo, la financiación de la cooperación internacional que encabezaba USAID se concentró en los denominados "grupos de riesgo", actualmente la intervención sigue enfocada a los mismos grupos, sin embargo ahora avalada por los estudios "científicos" realizados en estos últimos años, - por expertos locales - que a través de los datos numéricos aseguran que la epidemia en Bolivia está concentrada en las trabajadoras sexuales, hombres que tienen sexo con hombres y trans.

No hay duda de que el dato cuantitativo y epidemiológico es importante. Sin embargo, obtener un cuestionario acorde con la realidad sociocultural supone sumar dichos datos a una investigación cualitativa más amplia y no a priori. De ahí la importancia de realizar etnografías que puedan dar cuenta del por qué los programas de prevención no están funcionando, ya que en Bolivia el 50\% del total de personas notificadas al momento del diagnóstico son detectadas en caso SIDA y no en fase $\mathrm{VIH}^{16}$.

\section{A modo de conclusión}

De todo lo expuesto se puede concluir que las investigaciones, financiadas por las agencias globales sobre la problemática del VIH/SIDA en Bolivia, responden no sólo a aspectos técnicos, sino fundamentalmente ideológicos. Estos aspectos han influido en la recolección de los datos y en el análisis de los mismos. Las investigaciones se han basado en un manual creado en el exterior y que ha contribuido a la homogeneización de grupos: trabajadoras sexuales, HSH y GBT, ignorando las heterogeneidades internas a estos mismos grupos y negando la expansión de la epidemia en la población general.

En la mayoría de las investigaciones a las que hemos hecho referencia, los "comportamientos" sexuales se han considerado de manera generalizada como si fueran homogéneos o equivalentes en todo el país. Fundamentalmente, a partir de estudios cuantitativos o, en su caso, de estudios denominados cualitativos, aun cuando en su esencia se tratan como estudios cuantitativos, debido al escaso tiempo en la recogida de los datos, y a que el análisis se redujo a la contabilización de los casos y los "comportamientos" y no a la descripción y análisis de las prácticas y representaciones sociales.

Tanto el concepto de comportamiento como el de conocimiento, involucran aspectos más amplios - socioculturales, económico-políticos e ideológicos - que no pueden ser abordados en exclusividad a través de un enfoque psicológico. A priori y desde afuera se ha decidido qué conocimientos deben tener los grupos, sin que se deje espacio a la aparición del dato que se ignora, el dato que surge cuando no se tiene un esquema predeterminado para la recolección de los mismos y cuando se problematiza la realidad desde lo que se desconoce y no exclusivamente desde lo que se conoce o se cree que se conoce.

En muchas situaciones es la pobreza la causa final de la elevada mortalidad que se registra entre las personas que, en Bolivia, reciben tratamiento antirretroviral. El VIH/SIDA no puede ser descontextualizado de la situación socioeconómica del país ni de las condiciones de vulnerabilidad que posee la salud pública. Aspecto que ya ha sido mencionado por otros autores para diversos países de la región ${ }^{17-19}$.

La población boliviana se caracteriza por ser en su gran mayoría indígena, circunstancia que diferencia claramente al país en relación a otros. Al obviarse esta categoría étnica, por un lado se evita asociar la patología con determinados grupos sociales aunque por otro, supone ocultar la dispersión de la epidemia entre la población general. En este contexto, resulta fundamental definir el concepto de población general, así como conocer las formas de entender y atender el VIH por parte de la totalidad de las poblaciones que habitan en el país. Bolivia cuenta con 36 pueblos indígenas diferentes, además de poblaciones mixtas, criollos, mestizos, etc. 
Si bien el Ministerio de Salud ha contribuido a lo largo de los últimos años, con la ayuda de la cooperación internacional, al mejoramiento de las condiciones de los CDVIR, no se ha incidido, sin embargo, en su eliminación ni en el cuestionamiento ideológico y político sobre lo que representan.

Las investigaciones están concebidas a fin de que aparezca el dato que previamente se desea constatar, aquel que justifica la intervención planificada, sin que se cuestione ningún otro aspecto. Encuentro que no sólo existen problemas en el instrumento de recolección de los datos sino también en el análisis e interpretación de los mismos, debido a que no hay una base epistemológica, ni discusión teórica previa que permita comprender por qué una variable se comporta de una forma y no de otra en la población de estudio. Las estrategias de investigación que proponen las agencias globales y las razones por las cuales se toman esas estrategias deberían formar parte del debate y de la reflexión teórica.

La debilidad metodológica de las investigaciones realizadas y la falta de rigurosidad, sugiere también un problema de formación de los investigadores, problemática que si bien es del ámbito educativo; se refleja y afecta al de salud. La au- sencia de métodos y enfoques adecuados y de actitud crítica es un problema central de falta de formación apropiada, a lo que hay que añadir la existencia de cada vez menos investigadores que no estén financiados por las agencias globales, que puedan realizar críticas profundas al modelo de investigación imperante en los RAP.

Comparto con Standing ${ }^{20}$ que es necesario el conocimiento contextual de producción bibliográfico, antes de adoptarlo como una fuente de información real. Y que los significados socialmente específicos sobre sexualidad son más importantes en términos de riesgo que el número de parejas con las que tiene relaciones una persona.

Si todo el esfuerzo se centra en cuantificar los enfermos de VIH/SIDA y a aquellos que reciben tratamiento, se están dejando de lado los problemas centrales de la epidemia, problemas que, sin duda, no son los de la mera cuantificación. La exclusiva cuantificación de todo aquello que se refiere a "comportamientos", supone abandonar la reflexión en torno a las falencias metodológicas, teóricas y epistémicas de las investigaciones y los estudios que se efectúan a fin de propiciar el diagnóstico o con la voluntad de evidenciar la situación de la epidemia y la de las personas que conviven con el virus.

\section{Agradecimientos}

Quiero dar un especial agradecimiento a todo el personal sanitario que facilitó mi estadía en los servicios de salud y a las PVVS y familiares que compartieron sus historias. A María Julia Castellón-Arrieta, Oriol Romaní y Javier Luna Orosco por sus acertados comentarios que siempre ayudan a enriquecer mis textos. Y al apoyo del Convenio de Desempeño Universidad de TarapacáMineduc. 


\section{Referencias}

1. Bolivia. Ministerio de Salud y Deportes. Dirección General de Servicios de Salud. Programa Nacional ITS/VIH/SIDA. Informe Nacional sobre los progresos realizados en la aplicación del UNGASS (20082009). La Paz: Ministerio de Salud y Deportes; 2010.

2. Bolivia. Ministerio de Salud y Deportes. Unidad Nacional de epidemiología. Programa Nacional ITS/ VIH/SIDA. UNGASS Bolivia, 2006-2007. Informe sobre la respuesta Nacional a la Epidemia del VIH/ SIDA en Bolivia. La Paz: Ministerio de Salud y Deportes; 2007.

3. Ramírez Hita S. Calidad de atención en salud. Prácticas y representaciones sociales en las poblaciones quechuas y aymaras del altiplano boliviano. La Paz: OPS, OMS; 2010.

4. Ramírez Hita S. Salud Intercultural. Crítica y problematización a partir del contexto boliviano. La Paz: ISEAT; 2011.

5. Ramírez Hita S. La contribución del método etnográfico en el registro del dato epidemiológico. Epidemiología sociocultural indígena quechua de la ciudad de Potosí. Revista Salud Colectiva 2009; 5(1):63-85.

6. Bolivia. Ministerio de Salud y Deportes. Informe de monitoreo y evaluación de la implementación del compromiso UNGASS sobre el VIH/SIDA en Bolivia. La Paz: Ministerio de Salud y Deportes; 2006. (Serie Documentos de Divulgación Científica. UNAIDS)

7. Bolivia. Ministerio de Salud y Deportes. Plan estratégico del Programa Nacional de ITS/VIH/SIDA para la prevención y control del ITS/VIH-SIDA. 20062010. La Paz: Ministerio de Salud y Deportes; 2006.

8. Brown T. AIDS, risk and social governance. Soc Sci Med 2000; 50(9):1273-1284

9. Frankenberg R. The impact of HIV/AIDS on concepts relating to risk and culture within British community epidemiology: candidates or targets for prevention. Soc Sci Med 1994; 38(10):1325-1335.

10. Glick N, Crystal S, Lewellen D. Risky business: the cultural construction of AIDS risky groups. Soc Sci Med 1994; 38(10):1337-1346.

11. Sassen S. Una sociología de la globalización. Buenos Aires: Katz Editores; 2007.

12. Family Health International. Encuestas de Vigilancia del Comportamiento. Directrices para encuestas del comportamiento repetidas en poblaciones de riesgo al VIH. Durham: USAID, DFID, FHI; 2000.
13. ONUSIDA. Seguimiento de la Declaración de compromiso sobre VIH/sida. Directrices para el desarrollo de indicadores básicos. Ginebra: ONUSIDA; 2009.

14. Bolivia. Ministerio de Salud y Deportes. Programa Nacional de ITS/VIH/SIDA. Estudio sobre Sexualidad Masculina y VIH en Bolivia. La Paz: Ministerio de Salud y Deportes; 2009.

15. Ramírez Hita S. Ética y calidad en las investigaciones sociales en salud. Los desajustes de la realidad. Acta Bioética 2011; 17(1):61-71.

16. Proto J, Schaaf D, Suarez M, Darras C. Entorno epidemiológico y respuesta a la epidemia del VIH en Bolivia. Rev Panam Salud Pública 2008; 23(4):288294.

17. Parker R. Pobreza e HIV/AIDS aspectos antropológicos e sociológicos. Cad Saude Publica 2000; 16(Supl. 1): 89-102.

18. Aggleton P. Global priorities for HIV/AIDS intervention research. Int J STD AIDS 1996; 7(Supl. 2):1316.

19. Ayres Júnior CM. Epidemiologia sem números: Outras reflexões sobre a ciência epidemiológica, a propósito da AIDS. In: Seminário Epidemiologia Social da AIDS, Anais: 8-19. Rio de Janeiro: ABIA, IMS-UERJ; 1994.

20. Standing H. AIDS: Methodological and conceptual problems in the investigation of sexual behavior of Sub-Sahara Africa. Soc Sci Med 1992; 34(5):475-483.

Artigo apresentado em 20/03/2012

Aprovado em 29/05/2012

Versão final apresentada em 20/06/2012 